\title{
Diet modeling showed that local foods can secure nutrient adequacy except for iron in 19-30 years urban Egyptian women
}

\author{
Chloé Brouzes ${ }^{1}$, Nicolas Darcel $^{1}$, Daniel Tomé ${ }^{1}$, Sanaa Youssef Shaaban ${ }^{2}$, \\ Yasmin Gamal El Gendy ${ }^{2}$, Hisham Khalil ${ }^{3}$, Elaine Ferguson ${ }^{4}$ and Anne Lluch $^{5}$ \\ ${ }^{1}$ UMR PNCA, AgroParisTech, INRA, Université Paris-Saclay, Paris, France, \\ ${ }^{2}$ Pediatric and Clinical Nutrition Department, Faculty of Medicine, Ain Shams University, Cairo, Egypt, \\ ${ }^{3}$ Danone Nutricia Egypt, Cairo, Egypt, \\ ${ }^{4}$ Department of Population Health, London School of Hygiene and Tropical Medicine, London, United Kingdom and \\ ${ }^{5}$ Danone Nutricia Research, Centre Daniel Carasso, Palaiseau, France
}

\begin{abstract}
Growing urbanization and rapid changes in dietary patterns and lifestyle led to a nutrition transition in several Arabic countries, including Egypt. While Egyptian women have one of the highest mean body mass index worldwide, they also suffer from several micronutrient deficiencies, more particularly iron and vitamin D. The objective of this study was to identify changes needed in dietary practices and product offer, which could help to rebalance energy and nutrient intakes for women living in urban Egypt. Food intakes were obtained from a 4-days dietary record in 130 women aged 19-30 years from urban Egypt. Food prices were collected in modern and traditional trades typical from middle socio-economic classes in Cairo to calculate diet cost. Modeling analyses (with Optifood software) were used to identify problem nutrients and design affordable food-based recommendations (FBRs). The study assessed whether the most consumed foods (i.e. consumed by $>5 \%$ of women) could theoretically ensure nutrient adequacy under the mean diet cost, without exceeding recommendations in energy, SFAs, sugars and sodium. The potential of fortified foods to improve intakes of the most problematic micronutrient to cover was tested in additional modeling analyses. Preliminary results from modeling analyses indicate that iron appeared as the most limiting nutrient with locally consumed foods. Daily consumption of fruits, vegetables, milk or yoghurt, and tahini (sesame paste) associated with specific food choices in the meat-fish-eggs category would result in a low percentage of women at risk of inadequate intakes for 11 out of 12 modeled micronutrients. Among the fortified foods tested, iron fortified bread, rice, milk or yoghurt are the most promising vectors. Local and consumed foods allow to meet nutrient adequacy for most nutrients except iron, for which fortified products would be required. Tailored strategies are needed to promote acceptable FBRs (and fortified products) and secure that nutrient requirements are met by most Egyptian women.
\end{abstract}

\section{Conflict of Interest}

Danone Research has funded the study. 